\title{
A Study of the Structural Stability of TIC, TIN and TiO in the Pressure Range up to 65 GPa Using Synchroton Radiation*
}

\author{
Axel Nørlund Christensen, ${ }^{a}$ Leif Gerward, ${ }^{b, \dagger}$ Janus Staun Olsen ${ }^{c}$ and Stig Steenstrup ${ }^{c}$ \\ aDepartment of Inorganic Chemistry, Aarhus University, DK-8000 Aarhus C, 'Laboratory of Applied Physics, Technical University of \\ Denmark, DK-2800 Lyngby, and 'Physics Laboratory, University of Copenhagen, H. C. Ørsted Institute, Universitetsparken 5, \\ DK-2100 Copenhagen, Denmark
}

\begin{abstract}
Christensen, A. N., Gerward, L., Olsen, J. S. and Steenstrup, S., 1990. A Study of the Structural Stability of TiC, TiN and TiO in the Pressure Range up to $65 \mathrm{GPa}$ Using Synchroton Radiation. - Acta Chem. Scand. 44: 752-754.
\end{abstract}

The titanium compounds TiC, TiN and TiO are cubic with the $\mathrm{NaCl}$ type structure (space group $\mathrm{Fm} \overline{3} \mathrm{~m}$ ). The lattice constant decreases monotonically along the series $\mathrm{TiC}$, TiN and $\mathrm{TiO}$, together with a decreasing radius of the nonmetal atom. These compounds have a wide range of nonstoichiometry. TiC and TiN have mainly vacancies on the carbon and nitrogen sublattices, respectively. TiO has vacancies on the titanium as well as the oxygen sublattices. The vacancies are arranged in a short-range order that upon annealing can result in a long-range order of the occupied and vacant sites in the metal and non-metal sublattices. In the case of $\mathrm{TiO}$ this results in a monoclinic unit cell. The non-stoichiometry of the compounds makes the cubic unit cell parameter dependent on the composition $x$, corresponding to the chemical formulae $\mathrm{TiC}_{x}, \mathrm{TiN}_{x}$ and $\mathrm{TiO}_{x}$.

Structural phase transformations in $\mathrm{TiC}, \mathrm{TiN}$ and $\mathrm{TiO}$ could possibly occur at high pressure because of the nonstoichiometric nature of these compounds. The $\mathrm{NaCl}$ structure could then possibly transform to the $\mathrm{CsCl}$ structure, in which case the coordination number would increase from six to eight. This is in agreement with the general rule that the coordination number increases with pressure. The present work was undertaken in order to investigate possible structural phase transformations in $\mathrm{TiC}, \mathrm{TiN}$ and $\mathrm{TiO}$ in the pressure range up to $65 \mathrm{GPa}(=650 \mathrm{kbar})$.

\section{Experimental}

X-Ray powder diffraction spectra were recorded at HASYLAB-DESY, Hamburg, FRG, using synchrotron radiation and the white-beam energy-dispersive method. Experimental details have been published elsewhere. ${ }^{1}$ The electron energy of the storage ring DORIS II was $3.7 \mathrm{GeV}$ in a run

\footnotetext{
* Paper presented at the XIII Nordiske Strukturkemikermøde, Copenhagen, Denmark, January 8-10, 1990.

$\dagger$ To whom correspondence should be addressed.
}

dedicated to synchrotron radiation work. High pressure was achieved in a diamond anvil cell, where the sample and a small ruby chip was inclosed in small hole (diameter 0.1 $\mathrm{mm}$ ) in an Inconel gasket. A 4:1 methanol:ethanol mixture was used as the pressure transmitting medium. The pressure was determined from the wavelength shift of the red ruby line using the nonlinear pressure scale of Mao et al. ${ }^{2}$

Diffraction lines from the gasket were avoided by reducing the incident beam cross-section to $0.06 \mathrm{~mm} \times 0.06 \mathrm{~mm}$ using two pairs of crossed slits. The lattice constant at atmospheric pressure was determined from a zero-pressure spectrum with the sample situated in the diamond anvil cell. The Bragg angle associated with the diffraction spectrum was found from another zero-spectrum, where the sample was replaced by a $\mathrm{NaCl}$ powder with a known lattice constant. All experiments were performed at room temperature.

\section{Results and discussion}

The unit cell constants were refined by a least-squares method using nine diffraction lines, recorded in an energy range from 20 to $57 \mathrm{keV}$ for a Bragg angle of $7.2^{\circ}$. All diffraction spectra of $\mathrm{TiC}$, TiN and TiO could be indexed assuming the $\mathrm{NaCl}$ type structure. Thus, contrary to expectations, no structural phase transformations occur in the observed pressure range. In the case of TiN a slight distortion of the cubic unit cell was observed for pressures above about $20 \mathrm{GPa}$. This is illustrated in Fig. 1, where we have plotted the ratio between the observed spacings of the (111) planes and the (200) planes as a function of pressure. For a cubic lattice the theoretical value is 1.155 . As seen in Fig. 1 the observed value rises to about 1.16 above $20 \mathrm{GPa}$. The distortion is posssibly a preparatory stage for a phase transformation at a still higher pressure.

The variation of the unit-cell volume $V$, with pressure, $P$, can be described by the Murnaghan equation of state, ${ }^{3}$ 


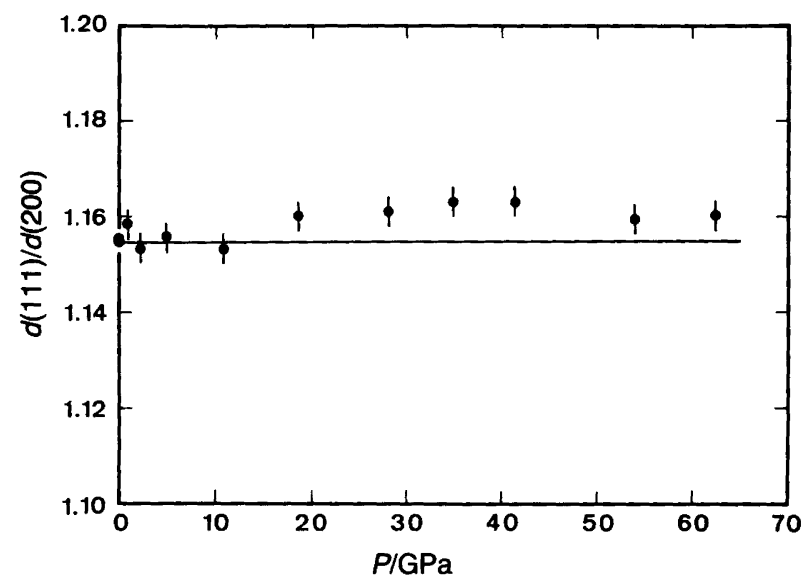

Fig. 1. Ratio between the spacings of the (111) planes and the (200) planes for TiN as a function of pressure. The horizontal line indicates the theoretical value $\mathbf{1 . 1 5 5}$ for a cubic lattice.

$P=\left(B_{0} / B_{0}{ }^{\prime}\right)\left[\left(V_{0} / V\right)^{B_{0}}-1\right]$

eqn. (1), where $V_{0}$ is the volume at zero pressure, $B_{0}$ the isothermal bulk modulus and $B_{0}{ }^{\prime}$ its pressure derivative, evaluated at zero pressure. Table 1 lists the $B_{0}$ and $B_{0}{ }^{\prime}$ values resulting from least-squares fits of the Murnaghan equation of state to the expermental pressure-volume data. Also included in Table 1 are the lattice constants, $a_{0}$, at atmospheric pressure, determined as described above. They agree within the experimental errors with the corresponding values given by Wyckoff: ${ }^{4} 4.319,4.235$ and 4.177 $\AA$ for $\mathrm{TiC}, \mathrm{TiN}$ and $\mathrm{TiO}$, respectively.

Concerning the pressure-volume relation, $\mathrm{TiO}$ is the most well-behaved sample of the three compounds. As seen in Fig. $2 c$ the Murnaghan equation of state describes the experimental data very well in the whole pressure range. Moreover, the pressure derivative of the bulk modulus is close to 4 , which is a normal value for most solids. Thus we have some confidence in the resulting value of 250 GPa for the bulk modulus, although it is $30 \%$ larger than the value of $182 \mathrm{GPa}$ given by Taylor and Doyle. ${ }^{5}$ It should also be noted that the pressure range in our case is ten times larger than in the previous determination.

The pressure-volume data for $\mathrm{TiC}$ and $\mathrm{TiN}$ are more scattered, and it has not been possible to fit to a single equation of state in the entire pressure range. Thus the $B_{0}$

Table 1. Lattice constant, $a_{0}$, bulk modulus, $B_{0}$, and its pressure derivative, $B_{0}{ }^{\prime}$, evaluated at zero pressure. The uncertainties, given in parentheses, are the standard errors of the leastsquares fits to the experimental data.

\begin{tabular}{llll}
\hline Compound & $a_{0} / \AA$ & $B_{0} / \mathrm{GPa}$ & $B_{0}{ }^{\prime}$ \\
\hline $\mathrm{TiC}$ & $4.328(9)$ & $220(30)$ & $12(4)$ \\
$\mathrm{TiN}$ & $4.237(2)$ & $300(40)$ & $7(3)$ \\
TiO & $4.181(4)$ & $250(10)$ & $4.0(4)$ \\
\hline
\end{tabular}
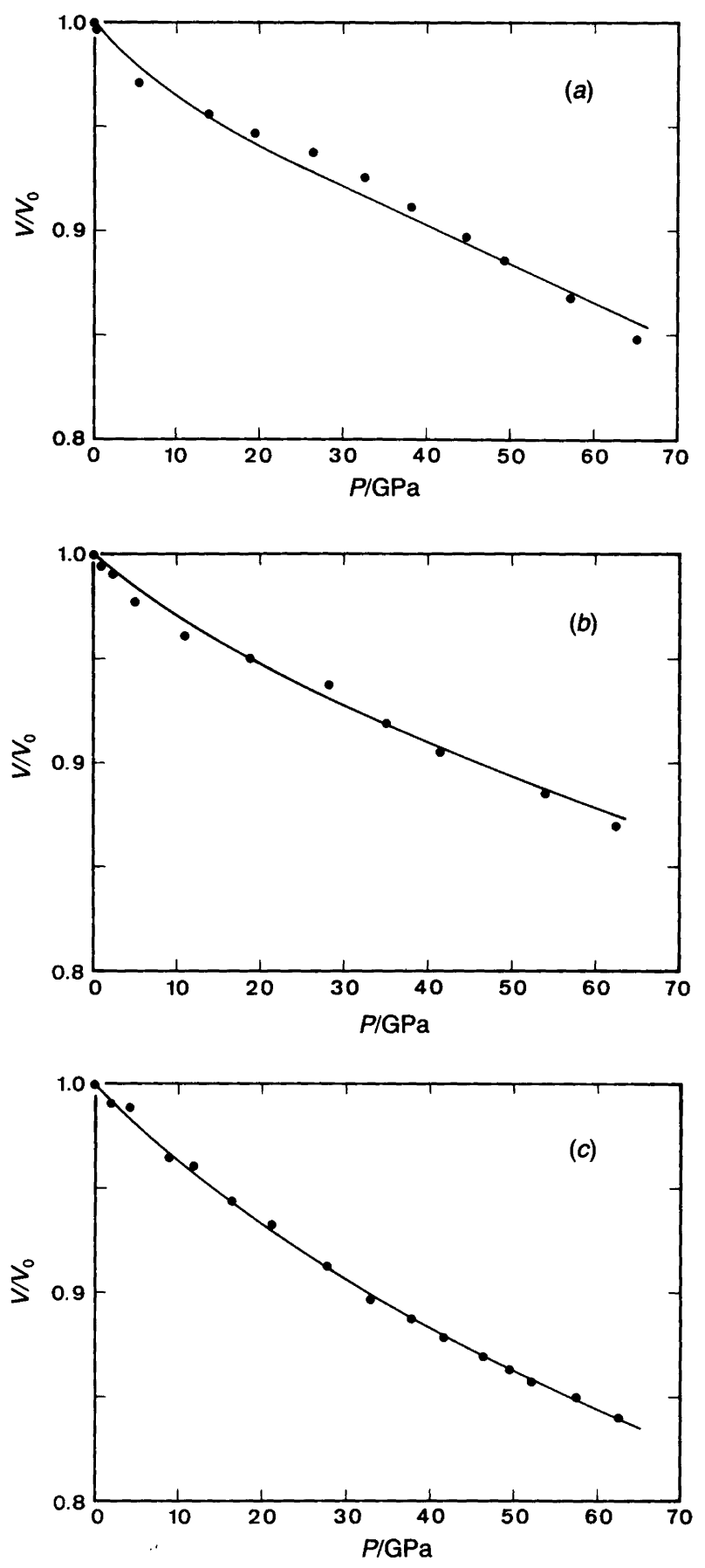

Fig. 2. Relative volumes as a functions of pressure. The full curves have been calculated from the equation of state using the data from Table 1. (a) TiC, (b) TiN, (c) TiO.

and $B_{0}{ }^{\prime}$ values given in Table 1 have been obtained in the pressure ranges up to $25 \mathrm{GPa}$ for $\mathrm{TiC}$ and up to $40 \mathrm{GPa}$ for TiN. Also the large $B_{0}{ }^{\prime}$ values are suspect. The irregular behaviour might be related to rearrangements of the vacancies in the samples at high pressure. The bulk moduli are in good agreement with the few published values available. For TiC Drickamer et al. ${ }^{6}$ quote $220 \mathrm{GPa}$ and Bridg$\operatorname{man}^{7}$ quotes $208 \mathrm{GPa}$. From elastic constants given by 


\section{SHORT COMMUNICATION}

Toth $^{8}$ one can calculate $240 \mathrm{GPa}$. For TiN Bridgman ${ }^{7}$ has measured a bulk modulus of $295 \mathrm{GPa}$.

In conclusion, we have found that $\mathrm{TiC}, \mathrm{TiN}$ and $\mathrm{TiO}$ retain their $\mathrm{NaCl}$ type structure in the pressure range up to $65 \mathrm{GPa}$. Bulk moduli are in good agreement with the few published values for $\mathrm{TiC}$ and $\mathrm{TiN}$, whereas our value for $\mathrm{TiO}$ is $30 \%$ larger than a previous determination.

Acknowledgements. We thank HASYLAB-DESY for permission to use the synchrotron radiation facility. Financial support from the Danish Natural Science Research Council is gratefully acknowledged.

\section{References}

1. Olsen, J. S., Benedict, U., Dabos, S., Gerward, L. and Itié, J.P. Physica B 144 (1986) 56.

2. Mao, H. K., Bell, P. M., Shaner, J. W. and Steinberg, D. J. J. Appl. Phys. 49 (1978) 3276.

3. Murnaghan, F. D. Finite Deformation of an Elastic Solid, Wiley, New York 1951.

4. Wyckoff, R.W.G. Crystal Structures, 2nd ed., Wiley, New York 1962, Vol. 1, p. 90

5. Taylor, A. and Doyle, N. J. In: Eyring, L. and O'Keefe, M., Eds., Chemistry of Extended Defects in Non-metallic Solids, North-Holland, Amsterdam 1970, p. 523.

6. Drickamer, H. G., Lynch, R. W., Clenenden, R. L. and PerezAlbuerne, E.A. Solid State Phys. 19 (1966) 144.

7. Bridgman, P. W. In: Samsonov, G. V., Ed., High Temperature Materials. No. 2, Properties Index, Plenum Press, New York 1964, p. 187

8. Toth, L. E. Transition Metal Carbides and Nitrides, Academic Press, New York 1971, pp. 146-149.

Received January 17, 1990. 